\title{
L'interleukine-21, \\ une cytokine clé dans le contrôle du VIH et d'autres \\ infections virales chroniques
}

> La différenciation et les fonctions effectrices des cellules immunes sont principalement contrôlées par les cytokines. L'infection par les virus entraîne souvent des dysfonctionnements de la réponse immunitaire. Ces dérèglements se manifestent par une sécrétion altérée des cytokines et une dérégulation de leurs effets biologiques. Parmi celles-ci, on trouve l'interleukine-2l (IL-2l), une cytokine récemment découverte qui exerce des effets multiples et pléïotropes sur les cellules immunes et joue un rôle indispensable dans le contrôle des infections virales aiguës et chroniques. Cette revue présente les connaissances actuelles concernant les effets biologiques de l'IL-2l sur la réponse immunitaire, et discute son rôle dans la mise en place de la réponse immune antivirale, et en particulier celle dirigée contre le VIH-l (virus de l'immunodéficience humaine). L'IL-21 est présentée comme un nouvel agent thérapeutique et un «adjuvant » potentiellement utilisable dans des stratégies de vaccination contre les infections virales. <

La réponse immunitaire est constituée de deux éléments principaux : l'immunité innée et l'immunité adaptative. Ces deux réponses interagissent de façon coordonnée dans l'organisme afin de contrôler les infections virales. Les composantes cellulaires de la réponse immune innée incluent les cellules dendritiques, les cellules natural killer (NK), les macrophages et les granulocytes. Un des phénomènes clés du système immunitaire inné est la production de cytokines pouvant réguler la réponse immune adaptative induite par les lymphocytes T et B. Ces cytokines permettent le développement de réponses effectrices qui sont spécifiques d'un antigène. Les cytokines produites par le système immunitaire, comme celles produites par les cellules T activées, vont donc permettre le développement de la réponse immune, augmenter ses fonctions effectrices et faciliter son expansion,
Alexandre Iannello, Ossama Allam, Suzanne Samarani, Ali Ahmad

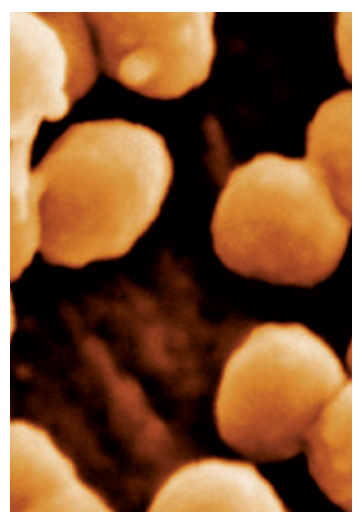

Laboratoire d'immunité innée, centre de recherche du centre hospitalo-universitaire ( $\mathrm{CHU})$ Sainte-Justine, département de microbiologie et d'immunologie, université de Montréal, 3175 chemin de la Côte Sainte-Catherine, Montréal, Québec H3T 1C5, Canada. alexandre.iannello@gmail.com ali.ahmad@rechercheste-justine.qc.ca

mais aussi induire une désactivation de la réponse lorsque les corps étrangers seront éliminés.

\section{L'interleukine-21}

L'interleukine-21 (IL-21) fait partie de la famille des cytokines de type I, une famille d'interleukines dont les récepteurs sont couplés à la chaîne gamma commune $\left(\gamma_{c} ;\right.$ CD132). Cette famille comprend notamment I'IL2, I'IL-4, I'IL-7, I'IL-9 et I'IL-15. L'IL-21 est principalement produite par les différents sous-types de lymphocytes T CD4 $4^{+}$, ainsi que les cellules NKT (natural killer $T$ cells) [1, 2]. Elle exerce ses effets biologiques en se liant à son récepteur (IL-2IR), ce qui entraîne la phosphorylation principalement de la protéine STAT-3 (signal transducer and activator of transcription-3) et faiblement des protéines STAT-1 et STAT-5 [2, 3]. L'IL-2IR est un hétérodimère constitué d'une chaîne $\alpha$ (IL-2IR $\alpha$ ) et d'une chaîne gamma commune $\left(\gamma_{c}\right)$. II est principalement exprimé par les cellules $T C D 4^{+}, C D 8^{+}$, les cellules $B$, les cellules dendritiques, les macrophages, les cellules NK et les kératinocytes [4-9]. L'IL-2l possède donc un rôle pléïotrope sur les différentes composantes de la réponse immunitaire (Tableau l). Cette cytokine joue un rôle essentiel, aussi bien dans la réponse antivirale induite par les cellules T et NK que dans la production d'anticorps de haute affinité par les cellules B (Figure 1). Du fait de son rôle important dans la différenciation et la maintenance des cellules Th17 (lymphocyte Thelper 17) [37], elle est aussi associée aux maladies auto-immunes et inflammatoires qui impliquent les cellules Th17, comme la maladie de Crohn [10]. 


\section{Lymphocytes B}

Aide à la formation des centres germinatifs Induit l'expression d'AID (activation-induced cytidine deaminase)

Permet une différenciation des cellules $B$ en plasmocytes et en cellules $B$ mémoires Induit leur apoptose lors d'une stimulation mitogénique (lipopolysaccharide ou LPS)

Augmente l'efficacité des anticorps en modulant la glycosylation de leur partie Fc Induit l'apoptose des cellules B produisant des lg

\section{Lymphocytes T CD8*}

Augmente leur prolifération, survie et fonctionnalité

Empêche la différenciation terminale des cellules $T \mathrm{CD} 8^{+}$et préserve leur capacité proliférative en réponse aux antigènes

Empêche l'épuisement des cellules T CD8+ lors d'infections virales chroniques

Inhibe les effets de l'IL-7 et de l'IL-15

Induit l'expression de CD62L et CCR7 à la surface des cellules T CD8 $8^{+}$

\section{Lymphocytes Th 17}

Permet leur différenciation et leur maintenance

\section{Lymphocytes $T$ folliculaires}

Favorise leur différenciation et leur migration vers les centres germinatifs

\section{Lymphocytes $\mathrm{T}$ régulateurs}

Inhibe leur différenciation en empêchant l'expression de FoxP3 (forkhead box P3) sans augmenter leur prolifération

\section{Cellules NK}

Augmente leur différenciation, leur maturation et leur survie

Augmente leur fonctionnalité et leur cytotoxicité

Augmente leur production d'IFN- $\gamma, T N F-\alpha, I L-10$, perforine et granzyme B

\section{Cellules NKT}

Augmente leur prolifération et leur survie

Stimule la sécrétion d'IL-4 et d'IL-13

Augmente l'expression de granzyme B et favorise leur morphologie granulaire

\section{Macrophages}

Induit la production d'IL-8 et facilite le recrutement des neutrophiles

Augmente leur capacité phagocytaire et leur activation

\section{Cellules dendritiques}

Retarde leur maturation, favorisant ainsi la capture d'antigènes

Tableau I. Effets biologiques de l'IL-21 sur les cellules du système immunitaire.

\section{Rôle de I'IL-21 dans la réponse antivirale}

L'induction d'une réponse immunitaire impliquant les cellules T CD4 $4^{+}$et $\mathrm{CD}^{+}$en conjonction avec les autres composantes du système immunitaire est nécessaire pour contrôler les infections virales aiguës ou chroniques. Il est aujourd'hui admis qu'en l'absence d'intervention des cellules T CD4+, notamment dans la production de cytokines, la réponse des cellules T CD $8^{+}$est compromise [11-13], et ce même s'il existe une réponse $\mathrm{T} C D 8^{+}$lors de la phase initiale d'une infection virale chronique. Cette incapacité des cellules $\mathrm{T}$ CD8 ${ }^{+}$à développer leurs fonctions effectrices entraîne l'absence du contrôle de l'infection au cours du temps (Figure 2) [11-13]. Cependant, les éléments régulateurs clés produits par les cellules $\mathrm{T} \mathrm{CD}^{+}$et nécessaires à l'obtention d'une réponse antivirale complète et efficace étaient jusqu'à récemment, mal compris, voire inconnus.

Trois études récentes réalisées sur des modèles in vivo murins d'infection virale persistante par le lymphocytic choriomeningitis virus (LCMV) ont démontré le rôle clé de I'IL-21 dans la réponse antivirale [14-16]. Lors de ces études, les auteurs ont utilisé deux souches différentes de LCMV (clone 13 et Armstrong) qui possèdent une pathogénicité différente chez l'animal inoculé. L'injection de fortes doses de LCMV clone 13 aboutit à une persistance à long terme du virus, ce qui représente donc une infection virale chronique. En revanche, l'infection par le LCMV Armstrong est rapidement controlée lors de la phase aiguë de l'infection.

Selon ces travaux, les effets biologiques de I'IL-21 sont nécessaires pour empêcher la baisse d'efficacité des cellules T CD8 ${ }^{+}$permettant le contrôle de l'infection. L'IL-21 est produite de façon prédominante lors des phases précoces et tardives de l'infection par les cellules $\mathrm{T} \mathrm{CD}^{+}$spécifiques du LCMV, et cette cytokine maintient les activités effectrices des cellules $\mathrm{T} \mathrm{CD}^{+}$spécifiques du LCMV. En effet, l'infection de souris knock-out (KO) pour l'IL-2lR avec la forme immunosuppressive (clone 13) du LCMV entraîne une délétion prononcée des cellules $\mathrm{T} C D 8^{+}$spécifiques du virus aboutissant à la persistance de ce dernier avec une virémie élevée. Au contraire, ces souris n'ont aucune difficulté à résoudre l'infection lors de l'inoculation des souches Armstrong. Lors de l'utilisation de moelles osseuses chimères provenant de souris sauvages ou KO pour I'IL-2IR (après transplantations croisées de cellules médullaires), les cellules $\mathrm{T} \mathrm{CD}^{+}$spécifiques 


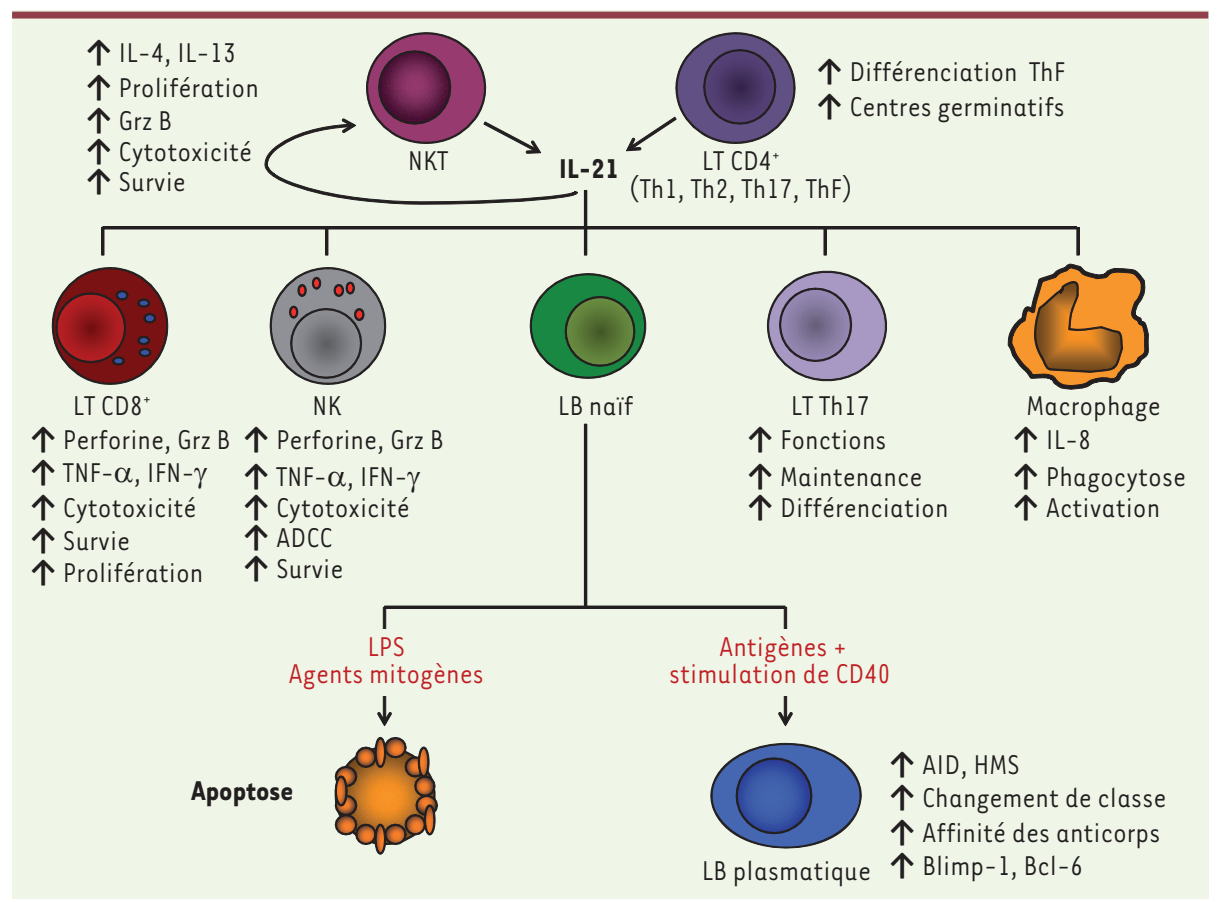

Figure 1. Effets biologiques de l'IL-21 sur les cellules du système immunitaire. L'IL-2l est principalement produite dans l'organisme par les cellules T CD4 ${ }^{+}$helper (Th1, Th2, Th17, ThF) et les cellules NKT. L'IL-21 joue un rôle positif ou négatif sur les cellules $B$ selon leur contexte d'activation. L'IL21 induit l'apoptose des cellules B activées de façon non spécifique par des agents mitogènes ou en présence de lipopolysaccharide (LPS), mais augmente leur différenciation lorsqu'elles sont activées de façon spécifique par un antigène et par des signaux costimulateurs des cellules $T$ (CD40/CD40L). Cette cytokine induit les facteurs de transcription (Blimp-1, Bcl-6) importants pour la différenciation en cellules plasmatiques ou mémoires. Elle augmente l'expression

d'AID (activation-induced cytidine deaminase), qui favorise le changement de classe des anticorps aboutissant à la production d'lgGl et lgG3 et des hypermutations somatiques (HMS), permettant la production d'anticorps de haute affinité. L'IL-2l possède des effets pro-inflammatoires sur les macrophages en induisant la sécrétion d'IL-8, qui permet notamment le recrutement des neutrophiles dans les tissus inflammatoires. L'IL-2l augmente l'activation des macrophages et la phagocytose, renforçant ainsi la présentation antigénique par ces cellules. L'IL-21 joue aussi un rôle important dans la différenciation et le maintien des cellules Th17, en induisant l'expression d'IL-23R à leur surface et le facteur de transcription ROR $\gamma t$ (retinoic acid receptor-related orphan receptor $\gamma$ ) qui est nécessaire au développement et au maintien de ces cellules. L'IL-2l augmente la survie et les fonctions effectrices des cellules T CD8+ ainsi que leur prolifération, en combinaison avec des cytokines comme l'IL-7 ou I'IL-15. D'une façon similaire, I'IL-21 augmente la prolifération des cellules NK en combinaison avec l'IL-2 ou l'IL-15 et augmente leur survie. દlle accroît la cytotoxicité de ces cellules et leur ADCC (antibody dependent cell cytotoxicity) en induisant la production de perforine, granzyme B (Grz B), IFN- $\gamma$ (interferon- $\gamma$ ) et TNF- $\alpha$ (tumor necrosis factor- $\alpha$ ). Finalement, l'IL-2l augmente la prolifération des cellules NKT lors d'une stimulation de leur CD3 en combinaison avec l'IL-2 ou l'IL-15. Elle stimule leur sécrétion d'IL-4 et d'IL-13, augmente leurs fonctions effectrices (Grz B) ainsi que leur survie. LB : lymphocyte B ; LT : lymphocyte T; ThF : lymphocyte T helper folliculaire; Grz B : granzyme B ; Bcl-6: B cell lymphoma protein-6; Blimp-1 : B lymphocyte-induced maturation protein-1; HMS : hypermutations somatiques.

du LCMV provenant des souris sauvages surpassent en fonction et efficacité ces mêmes cellules provenant de souris KO pour l'IL-2lR. L'injection directe d'IL-2l à des souris ne possédant pas de cellules $\mathrm{T} \mathrm{CD}^{+}$a permis de restaurer les fonctions antivirales exprimées par les cellules $T C D 8^{+}$ainsi que de réduire la charge virale chez ces souris [16]. L'IL-21 ne semble donc pas être nécessaire à la génération des cellules $\mathrm{T} \mathrm{CD8}^{+}$spécifiques du virus, mais elle serait importante pour la qualité de la réponse antivirale [16]. Ces études suggèrent le rôle indispensable de I'IL-2l au stade chronique de l'infection, plutôt qu'au stade de l'infection aiguë.

Cependant, des études plus récentes ont démontré que I'IL-21 est nécessaire lors des réponses primaire et secondaire [17]. Son rôle dans le contrôle des infections par le virus de l'hépatite $B$ et le virus de l'influenza a été aussi récemment documenté $[18,19]$. Ces études soulignent son rôle indispensable dans le contrôle de l'infection chronique, ainsi que le rôle clé joué par les cellules T CD4 ${ }^{+}$lors de la phase aiguë de l'infection et dans le développement la réponse antivirale primaire. Bien que le but premier de ces études n'était pas d'étudier l'impact de l'IL-2l sur la réponse humorale, une réduction significative de la production d'anticorps spécifiques du LCMV a été observée chez les souris KO pour I'IL-2lR suite à l'infection par la souche immunosuppressive (clone 13). Ces observations sont en accord avec le rôle clé joué par l'IL-2l dans la génération des plasmocytes et la production d'anticorps par ces derniers [3, 20]. Les souris KO pour I'IL-2IR produisent des anticorps de faible affinité ou non spécifiques du virus. En effet, des défauts dans la signalisation impliquant I'IL-21 au niveau des cellules $B$ affaiblissent la réponse des anticorps [21]. Ces données soulignent l'impact général et collectif de I'IL-2l dans la création et l'établissement de réponses immunitaires acquise et humorale lors d'une infection virale chronique. 


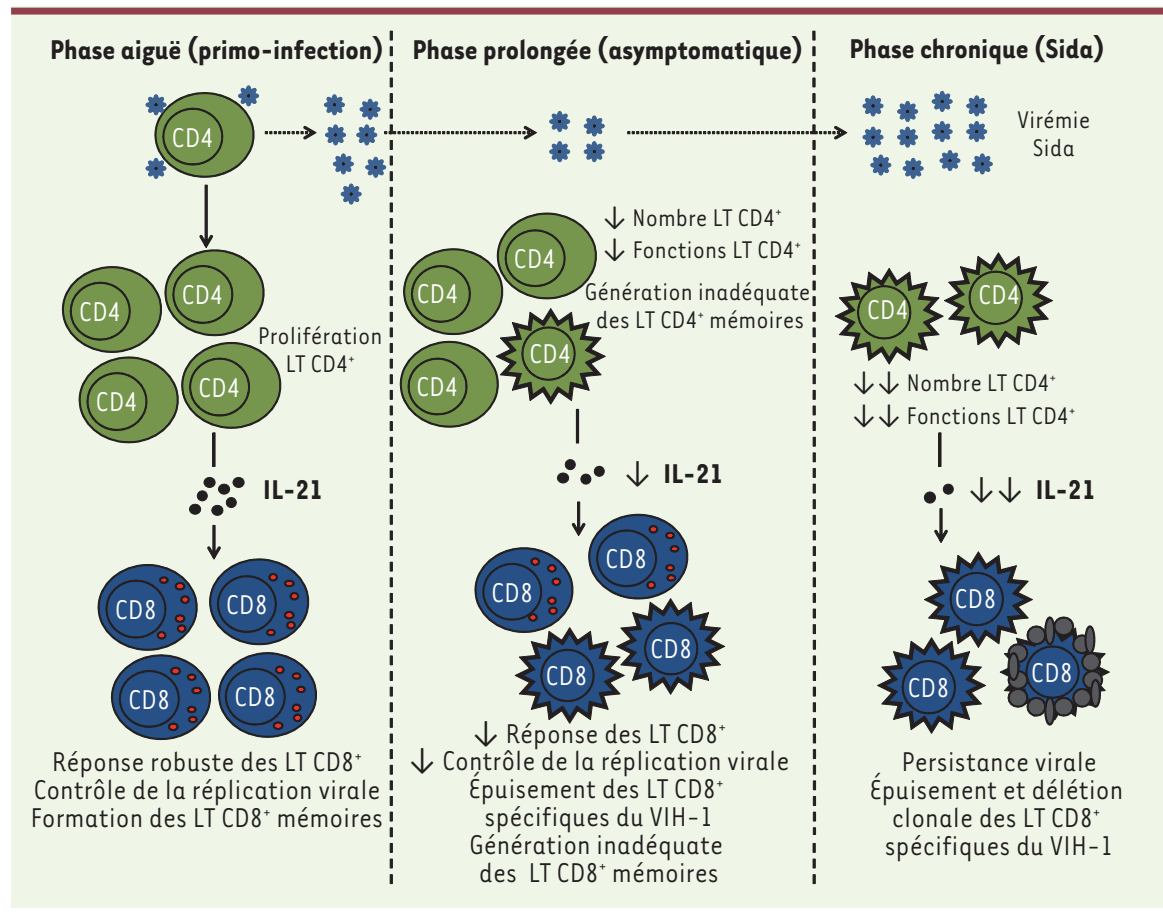

Figure 2. Importance de l'IL-2I produite par les cellules TCD4+ dans le contrôle des infections virales chroniques : l'exemple du VIH-1. La phase aiguë de l'infection par le VIH-l (primo-infection) induit la prolifération des cellules T CD4+ produisant des niveaux élevés d'IL-21. La sécrétion d'IL-21 permet une réponse robuste des cellules $T \mathrm{CD} 8^{+}$qui conduit à un contrôle de la réplication virale et à la formation de cellules T CD8 ${ }^{+}$mémoires. L'absence d'élimination totale du VIH-l par le système immunitaire amène, progressivement lors de la phase prolongée, à une baisse du nombre et de l'activité des cellules T CD4 et à une production inadéquate des cellules $\mathrm{TCD}^{+}$mémoires. Ceci aboutit à une baisse de la production d'IL-21 qui réduit progressivement la réponse impliquant les cellules $T$ CD $8^{+}$et la génération des cellules T CD $8^{+}$mémoires. Cette étape amorce une déplétion des cellules T CD $8^{+}$spécifiques du VIH-l et une perte de contrôle de l'infection. Lors de la phase chronique, l'augmentation de la réplication virale associée à la chute drastique du nombre des cellules T CD4 $4^{+}$produisant de l'IL-2l entraîne une persistance virale. On voit apparaître dans cette phase une déplétion sévère des cellules T CD $8^{+}$spécifiques du VIH-1, ainsi que leur délétion clonale qui aboutira finalement au développement du stade Sida.
d'IL-21, bien que cette restauration soit partielle [22]. Chez les patients non progresseurs à long terme qui sont capables de contrôler l'infection et le développement du SIDA sans traitement, les taux d'IL-2l circulante sont normaux [22]. Nos études démontrent que la capacité des lymphocytes T CD4 $4^{+}$des patients infectés à produire de l'IL-2l est réduite par rapport à celle des cellules provenant de patients non infectés. De plus, l'infection cause une déplétion des cellules T CD4 ${ }^{+}$spécifiques du VIH-l produisant I'IL-2l (Figure 2). L'infection directe des cellules $\mathrm{TCD}^{+}$réduit la production d'IL-2l en inhibant l'expression du facteur de transcription c-Maf [22]. En outre, les niveaux d'IL-2l chez les patients infectés sont corrélés à la polyfonctionnalité des cellules T CD8 ${ }^{+}$ spécifiques du VIH-1 [22]. Le rôle protecteur des cellules T $\mathrm{CD}^{+}{ }^{+}$spécifiques du VIH et produisant de I'IL-2I a aussi été documenté dans d'autres travaux [24, 25]. L'étude menée par Chevalier et al. [24] démontre que les cellules T CD4 ${ }^{+}$de patients « contrôleurs élites » produisent plus d'IL-2l lors d'une stimulation par des peptides du VIH-l que ceux qui expriment une virémie persistante et dont la maladie progresse. L'IL-21 produite par ces cellules augmente la production de perforine par les cellules T $\mathrm{CD}^{+}$spécifiques du VIH-l

\section{Rôle de I'IL-21 dans le contrôle de l'infection par le VIH-1}

Lors de l'infection par le VIH-l et le développement du syndrome d'immunodéficience acquise (Sida), les défauts observés dans les fonctions, l'entretien ainsi que la cohérence des cellules du système immunitaire sont en large partie attribués à une production altérée des cytokines et/ou à leur manque d'efficacité.

Nos études ont permis de montrer que les taux sériques d' IL-2l étaient considérablement réduits chez les patients infectés par le VIH-1 [22, 23]. Ces études ont été réalisées dans une large cohorte de patients au stade de la primo-infection, chez des patients chroniquement infectés avec ou sans traitement antirétroviral ainsi que chez différentes catégories de patients non progresseurs à long terme (aussi appelés « contrôleurs élites ») [38]. Nos études montrent que la production d'IL-2l est compromise assez tôt au cours de l'infection et que le traitement antiviral permet de restaurer les concentrations sériques provenant de patients chroniquement infectés dont la maladie progresse et augmente l'effet inhibiteur de ces cellules sur la réplication du VIH-1 [24]. Une étude a de plus récemment démontré que les cellules $\mathrm{T} C \mathrm{CD} 8^{+}$des patients « contrôleurs élites » sont aussi capables de produire de I'IL-21 lors d'une stimulation peptidique spécifique du VIH-1 [26]. II est important de noter ici que ces cellules ne produisent pas d'IL-21 habituellement. Ces études soulignent donc le rôle clé de l'IL-2l dans le contrôle de l'infection par le VIH-l et le développement du Sida. L'absence d'IL-2l et de son effet pléïotrope chez les patients infectés pourrait donc avoir des conséquences néfastes sur la réponse antivirale impliquant les différentes composantes du système immunitaire (Figure 3). 


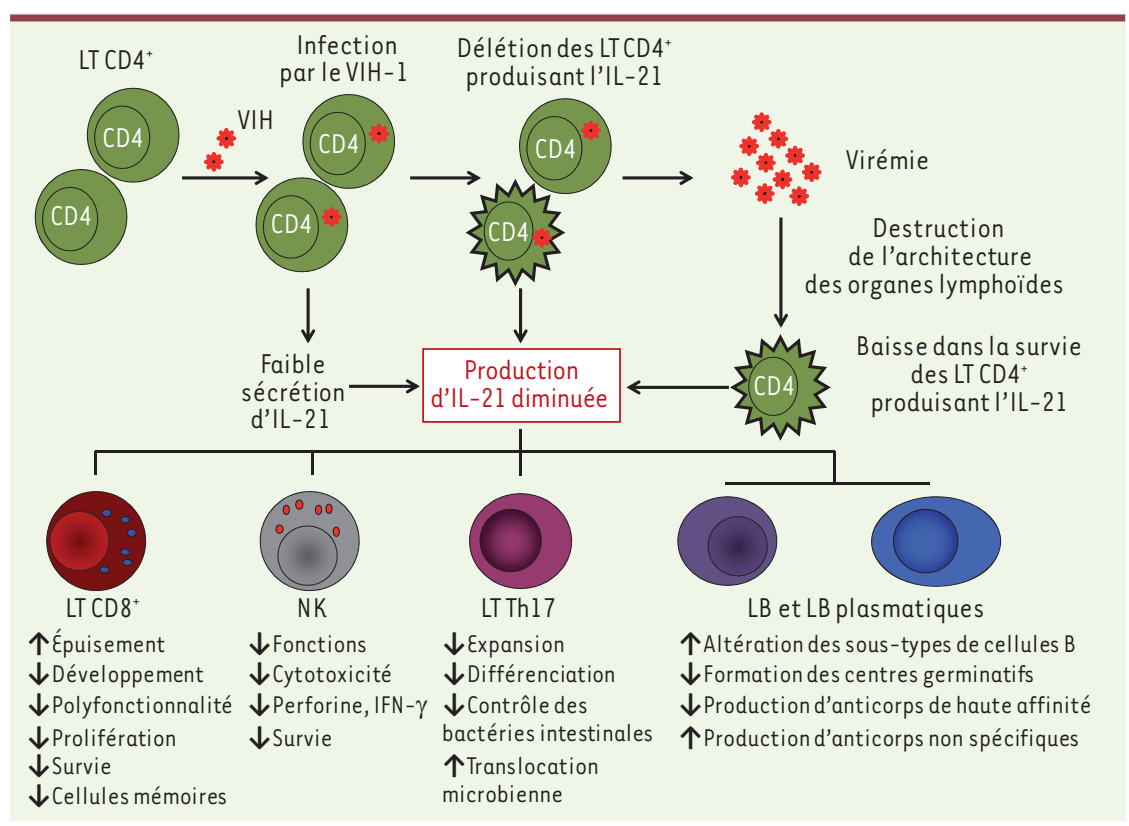

Figure 3. Conséquences des faibles niveaux d'IL-21 produits lors de l'infection par le VIH-1 sur le système immunitaire. L'infection des lymphocytes T CD4+ par le VIH-l réduit la production d'IL-21 au niveau de l'organisme par différents mécanismes. La réduction d'IL-2l est due à l'infection directe des cellules $\mathrm{T} \mathrm{CD4}^{+}$par le VIH-1, mais aussi à la perte de ces cellules lors du développement du Sida. Une production altérée d'IL-21 engendre des altérations des différents soustypes de cellules $B$ chez les patients infectés. L'absence d'IL-21 entraîne aussi une réduction de la formation des centres germinatifs qui jouent un rôle important dans la production d'anticorps de haute affinité contre les agents pathogènes comme le VIH-1. Une déficience en IL-21 entraîne la prolifération et une différenciation non spécifique des cellules B lors d'une stimulation par des agents mitogènes comme le LPS. Ceci aboutit à la production d'anticorps non spécifiques et inutiles au contrôle de l'infection, affaiblissant ainsi la réponse humorale de l'hôte. Au niveau des muqueuses, une réduction de la présence d'IL-2l affaiblit la différenciation et l'expansion des cellules Th17. Ceci pourrait entrâner un manque de contrôle de la flore intestinale et des translocations microbiennes qui vont promouvoir le développement du Sida. Les faibles niveaux d'IL-2l vont entraîner un épuisement des cellules T CD8 ${ }^{+}$, spécifiques du VIH-l et réduire leur développement en cellules mémoires. Les fonctions effectrices des cellules T CD8+, ainsi que celles des cellules NK qui permettent de contrôler la réplication virale et de contenir l'infection, seront affectées par l'absence d'IL-2l lors de l'infection par le VIH-1.

\section{L'IL-21 exogène, un nouvel outil pour renforcer la réponse immunitaire contre le VIH-1}

Plusieurs études ont montré le rôle de l'IL-2l dans le renforcement de la réponse antivirale. Nous avons observé que les sous-types majeurs de cellules NK exprimaient de façon constitutive des niveaux équivalents d'IL-21R [27]. L'expression de l'IL-21R n'est aucunement altérée chez les patients infectés par le VIH-1, quel que soit leur stade clinique et ce pour tous les différents sous-types de cellules NK. L'IL-2l augmente l'expression de perforine et d'IFN- $\gamma$, ainsi que la cytotoxicité exercée par les cellules NK, tant chez les personnes saines que celles qui sont infectées par le VIH-1 [27]. L'IL-21 n'accroît pas la prolifération des cellules NK lorsqu'elle est utilisée seule, mais elle augmente l'expression des protéines antiapoptotiques $\mathrm{Bcl}-2$ (B-cell lymphoma protein-2) et $\mathrm{Bcl}-\mathrm{X}_{\mathrm{L}}$ dans les cellules NK, et les cellules T. Elle augmente de ce fait la viabilité de ces cellules lors de cultures ex vivo [27]. L'IL-21 exerce des effets similaires sur les cellules T CD8 ${ }^{+}$de patients infectés par le VIH-1 [28].

Malgré leur effet positif sur l'immunité antivirale, le principal désavantage de l'utilisation thérapeutique des cytokines réside dans leur capacité à augmenter la réplication du VIH-1. Lors d'essais cliniques, l'administration d'IL-2 a permis d'accroître le taux de cellules T CD4 ${ }^{+}$ chez les patients infectés sans augmenter la réplication virale [29]. En comparant les effets de l'IL-21 à ceux de l'IL-2 et de l'IL-15 in vitro, nous avons observé que I'IL-21 n'amplifie pas la réplication du VIH-1 [27]. Nos résultats ont démontré que cette cytokine n'augmente pas la production de virus endogènes lorsqu'on l'ajoute à des cultures ex vivo prolongées de cellules mononucléées sanguines provenant de patients chroniquement infectés, virémiques, et sans thérapie antirétrovirale [27]. Une étude récente a démontré que l'injection d'IL-2l à des macaques Rhésus infectés par le virus d'immunodéficience simienne (VIS) augmente la fréquence et l'expression de perforine et de granzyme $B$ dans les cellules $T C D 8^{+}$mémoires et effectrices, tant au niveau périphérique qu'au niveau des ganglions lymphatiques mésentériques [30]. Cette augmentation a aussi été observée dans les cellules T $\mathrm{CD}^{+}$et les cellules NK périphériques. L'IL-2l accroît la fréquence des cellules $T$ CD8 $8^{+}$spécifiques du VIS exprimant l'IFN- $\gamma$ et leur cytotoxicité, au niveau périphérique ainsi que dans les ganglions lymphatiques. De plus, le titre d'anticorps dirigés contre le VIS augmente après injection d'IL-21, ainsi que le nombre de cellules T CD8 $8^{+}$mémoires. L'injection d'IL-2l a été bien tolérée, n'a pas augmenté la charge virale et n'a pas engendré d'activation cellulaire aberrante qui pourrait augmenter la réplication virale [30].

\section{L'IL-21, un « adjuvant 》 de la vaccination contre les infections virales}

Utilisant un modèle murin d'infection par le Herpes simplex virus-1 (HSV-1), Rodrigues et al. [31] ont montré que I'IL-2l augmente la fréquence des cellules 
1. Nécessaire au développement de la réponse antivirale cellulaire primaire et secondaire, ainsi qu'à la réponse humorale

2. Augmente la survie et la prolifération des cellules T et NK en modulant l'expression des protéines antiapoptotiques de la famille $\mathrm{Bcl}-2$

3. Augmente les fonctions effectrices des cellules T, NK et NKT

4. Empêche l'épuisement des cellules T CD8 ${ }^{+}$spécifiques du virus et augmente leur développement à long terme

5. Inhibe l'expression de TRAIL, empêchant ainsi la délétion dépendante de TRAIL des cellules T CD $8^{+}$spécifiques du virus

6. Inhibe la différenciation des cellules T régulatrices et augmente la résistance des cellules T spécifiques du virus à ces dernières

7. Permet de promouvoir la production d'anticorps spécifiques du virus et d'améliorer leur affinité

8. Induit l'apoptose des cellules B stimulées par du LPS ou des agents mitogènes, empêchant ainsi la production d'autoanticorps et d'anticorps non spécifiques lors de l'infection

Tableau II. Rôle de l'IL-21 dans la réponse immunitaire antivirale. Trail : TNFrelated apoptosis-inducing ligand.

T CD8 ${ }^{+}$spécifiques du virus [31]. L'injection de plasmides codant pour I'IL-15 et I'IL-21 a permis d'accroître le nombre de cellules T CD8 ${ }^{+}$ spécifiques des antigènes du HSV-l qui produisent de l'interféron- $\gamma$ $($ IFN- $\gamma$ ) lors de la phase aiguë de l'infection. Lors de l'infection chronique, l'injection de plasmides codant pour l'IL-15 et I'IL-21, seuls ou en combinaison, entraîne l'accroissement du nombre de cellules $T$ CD $8^{+}$produisant de l'IFN- $\gamma$ et du tumor necrosis factor- $\alpha$ (TNF- $\alpha$ ). De plus, l'association de (ou des) ADN plasmidique(s) codant pour ces cytokines à des antigènes viraux permet d'augmenter la prolifération et la polyfonctionnalité des cellules T CD8 ${ }^{+}$. Lors d'une autre étude, la co-injection intravasculaire à des souris infectées par le HSV-l d'un ADN plasmidique codant pour la glycoprotéine B du HSV-l et d'un plasmide codant pour l'IL-2l a augmenté le taux de survie des animaux [32]. Le nombre de lymphocytes $T$ cytotoxiques spécifiques de la glycoprotéine $B$, ainsi que la production d'anticorps neutralisants dirigés contre ce virus, ont été accrus. D’une façon similaire, l'injection intraoculaire de nanoparticules contenant des molécules d'ADN codant pour la glycoprotéine D du HSV-1 et d'IL-2l permet de conférer une protection contre l'infection par ce virus aux souris [33].

Des études ont également montré que l'IL-2l favorise l'expansion clonale de cellules T CD4 ${ }^{+}$spécifiques du human cytomegalovirus (HCMV) lors d'une stimulation peptidique [34]. De plus, elle augmente l'expression du récepteur CCR7 ( $C$-C chemokine receptor type 7 ) en préservant son activité fonctionnelle. D'une manière similaire, des études récentes ont montré que la stimulation de cellules $T$ CD $8^{+}$spécifiques du HCMV par des peptides viraux en combinaison avec de l'IL-21 permet d'induire la réexpression du CCR7 dans ces cellules, ce qui leur permet de répondre à une stimulation par des chimiokines [35]. Ces données suggèrent donc que la stimulation antigénique combinée à I'IL-2l peut induire la migration des cellules $T$ CD $8^{+}$au niveau des sites inflammatoires critiques lors des infections virales. Par conséquent,
I'IL-21 joue un rôle important dans la réponse immune dans une situation de réinfection ou de réactivation de virus latents.

Une étude in vivo a démontré que I'IL-2l, seule ou en combinaison avec I'IL-15, est capable de réguler la réponse immunitaire contre la glycoprotéine d'enveloppe (Env) du VIH-1 [36]. L'IL-2l a permis d'établir une résistance soutenue à la réplication et à la transmission du virus. L'IL-2l et l'IL-15 ont agi de façon synergique pour augmenter la réponse immunitaire de type mémoire. Ces deux cytokines ont permis de promouvoir l'expansion des cellules T CD8 ${ }^{+}$mémoires spécifiques des glycoprotéines d'enveloppe. Le traitement par l'IL-21 et I'IL-15 a augmenté les fonctions effectrices des cellules T CD8 $8^{+}$, permettant ainsi une protection efficace. De plus, ces deux cytokines sont capables d'accroître la cytotoxicité cellulaire dépendante des anticorps et la lyse induite par le complément ciblant des cellules exprimant les glycoprotéines d'enveloppes du VIH-1, suite à l'augmentation du niveau de production d'anticorps neutralisants spécifiques de ces protéines [36].

\section{Conclusions et perspectives}

Les mécanismes permettant de comprendre le développement de la réponse antivirale sont encore de nos jours d'un intérêt primordial. La difficulté de compréhension de ces mécanismes s'explique par la complexité des facteurs viraux agissant lors de l'infection, des mécanismes immunitaires impliqués dans leur contrôle, et en particulier ceux qui impliquent les cytokines. La compréhension de la biologie des cytokines et de leur importance dans la réponse antivirale permettra le développement de nouvelles stratégies thérapeutiques. Les différentes études mentionnées dans cette synthèse soulignent l'importance de l'IL-2l aux différentes étapes de la réponse antivirale. Le fait que cette cytokine agisse de façon pléïotrope sur les différents acteurs de la réponse immunitaire nous amène à proposer cette cytokine comme le ou l'un des facteurs clés fourni par les cellules T CD4 ${ }^{+}$pour orchestrer la réponse immunitaire antivirale et promouvoir ses fonctions effectrices (Tableau II). Une bonne compréhension de l'effet de I'IL-2l sur les différents compartiments du système immunitaire aura un impact majeur sur les stratégies thérapeutiques au niveau clinique. Cependant, ces stratégies thérapeutiques se doivent d'être mesurées et très contrôlées, car il est bien connu que les cytokines peuvent induire des effets complètement contraires à ceux recherchés si elles ne sont pas utilisées de façon appropriée. Sachant que l'IL-2l semble être bien tolérée par l'organisme, son utilisation thérapeutique chez des patients souffrant d'infections virales chroniques ou en 
tant qu' « adjuvant » vaccinal devrait être considérée comme une piste sérieuse dans un futur proche. $\diamond$

\section{SUMMARY}

Interleukin-21: a key cytokine for controlling HIV and other chronic viral infections

The differentiation, homeostatic proliferation and effector functions of different immune cells are controlled, to a large extent, by cytokines. Viruses often cause immune response dysfunctions by causing defects in the cytokine networks. The defects are often manifested by altered cytokine secretion and/or responsiveness to the cytokine. Among these cytokines, Interleukin-2l (IL-2l) is a relatively recently discovered cytokine, which is mainly produced by $C D 4^{+} T$ cells in the body, and exerts multiple and pleiotropic effects on various immune cells. Recent studies have shown that the cytokine is indispensable for controlling chronic viral infections. This review summarizes current knowledges concerning the biological effects of this cytokine on different components of the immune system. We also discuss how it contributes toward mounting efficient antiviral immunity and controlling chronic viral infections, especially HIV-1. The IL-1 cytokine represents a novel therapeutic agent for virus-infected patients as well as an adjuvant in antiviral vaccination strategies. $\diamond$

\section{LIENS D'INTÉRÊT}

Les auteurs déclarent n'avoir aucun lien d'intérêt concernant les données publiées dans cet article.

\section{RÉFÉRENCES}

1. Coquet JM, Kyparissoudis K, Pellicci DG, et al. IL-21 is produced by NKT cells and modulates NKT cell activation and cytokine production. J Immunol 2007 ; 178 : 2827-34.

2. Parrish-Novak J, Dillon S, Nelson A, et al. Interleukin 21 and its receptor are involved in NK cell expansion and regulation of lymphocyte function. Nature $2000 ; 408: 57-63$.

3. Spolski R, Leonard WJ. Interleukin-21: basic biology and implications for cancer and autoimmunity. Annu Rev Immunol 2008 ; $26: 57-79$.

4. Brandt K, Bulfone-Paus S, Foster DC, Ruckert R. Interleukin-21 inhibits dendritic cell activation and maturation. Blood $2003 ; 102: 4090-8$.

5. Caruso R, Fina D, Peluso I, et al. IL-21 is highly produced in Helicobacter pylori-infected gastric mucosa and promotes gelatinases synthesis. J Immunol 2007 ; 178 : 5957-65.

6. Distler JH, Jungel A, Kowal-Bielecka 0, et al. Expression of interleukin-2l receptor in epidermis from patients with systemic sclerosis. Arthritis Rheum $2005 ; 52: 856-64$.

7. Jin H, Carrio R, Yu A, Malek TR. Distinct activation signals determine whether IL-21 induces B cell costimulation, growth arrest, or Bim-dependent apoptosis. J Immunol 2004 ; 173 : 657-65.

8. Ozaki K, Kikly K, Michalovich D, et al. Cloning of a type I cytokine receptor most related to the IL-2 receptor beta chain. Proc Natl Acad Sci USA 2000 ; 97 : 11439-44.

9. Parrish-Novak J, Foster DC, Holly RD, Clegg CH. Interleukin-21 and the IL-21 receptor: novel effectors of NK and T cell responses. J Leukoc Biol $2002 ; 72: 856-63$.

10. Spolski R, Leonard WJ. The Yin and Yang of interleukin-2l in allergy, autoimmunity and cancer. Curr Opin Immunol $2008 ; 20: 295-301$.

11. Battegay M, Moskophidis D, Rahemtulla A, et al. Enhanced establishment of a virus carrier state in adult CD4 ${ }^{+}$T-cell-deficient mice. J Virol $1994 ; 68: 4700-4$.

12. Matloubian M, Concepcion RJ, Ahmed R. CD4 ${ }^{+} T$ cells are required to sustain CD8+ cytotoxic T-cell responses during chronic viral infection. J Virol 1994 ; $68: 8056-63$.

13. Ou R, Zhou S, Huang L, Moskophidis D. Critical role for alpha/beta and gamma interferons in persistence of lymphocytic choriomeningitis virus by clonal exhaustion of cytotoxic T cells. J Virol $2001 ; 75: 8407-23$.

14. Elsaesser H, Sauer K, Brooks DG. IL-21 is required to control chronic viral infection. Science 2009 ; $324: 1569-72$.

15. Frohlich A, Kisielow J, Schmitz I, et al. IL-21R on T cells is critical for sustained functionality and control of chronic viral infection. Science 2009 ; 324 : 1576-80.

16. Yi JS, Du M, Zajac AJ. A vital role for interleukin-21 in the control of a chronic viral infection. Science $2009 ; 324: 1572-6$.
17. Barker BR, Gladstone MN, Gillard G0, et al. Critical role for IL-21 in both primary and memory anti-viral CD8 ${ }^{+} \mathrm{T}$-cell responses. Eur J Immunol 2010 ; $40: 3085-96$

18. Publicover J, Goodsell A, Nishimura S, et al. IL-21 is pivotal in determining age-dependent effectiveness of immune responses in a mouse model of human hepatitis B. J Clin Invest 2011 ; 121 : 1154-62.

19. Pallikkuth S, Pilakka Kanthikeel S, Silva SY, et al. Upregulation of IL-2I receptor on B cells and IL-2l secretion distinguishes novel $2009 \mathrm{HINl}$ vaccine responders from nonresponders among HIV-infected persons on combination antiretroviral therapy. J Immunol 2011 ; 186: 6173-81.

20. Rankin AL, MacLeod H, Keegan S, et al. IL-21 receptor is critical for the development of memory B cell responses. J Immunol 2011 ; 186 : 667-74.

21. Avery DT, Deenick EK, Ma CS, et al. B cell-intrinsic signaling through IL-2l receptor and STAT3 is required for establishing long-lived antibody responses in humans.J Exp Med 2010 ; 207 : 155-71.

22. Iannello A, Boulassel MR, Samarani $S$, et al. Dynamics and consequences of IL-2l production in HIV-infected individuals: a longitudinal and crosssectional study. J Immunol $2010 ; 184$ : 114-26.

23. Iannello A, Tremblay C, Routy J, et al. Decreased levels of circulating IL-2] in HIV-infected AIDS patients: Correlation with $\mathrm{CD}^{+} \mathrm{T}$ cell counts. Viral Immunol $2008 ; 21: 385-8$.

24. Chevalier MF, Julg B, Pyo A, et al. HIV-1-specific interleukin-21+CD4+ T cell responses contribute to durable viral control through the modulation of HIV-specific CD8 ${ }^{+}$T cell function. J Virol $2011 ; 85$ : 733-41.

25. Yue FY, Lo C, Sakhdari A, et al. HIV-specific IL-21 producing CD4 ${ }^{+}$T cells are induced in acute and chronic progressive HIV infection and are associated with relative viral control. I Immunol 2010 ; 185 : 498-506.

26. Williams LD, Bansal A, Sabbaj S, et al. Interleukin-21-producing HIV-1specific CD8 T cells are preferentially seen in elite controllers. J Virol 2011 ; $85: 2316-24$.

27. Iannello A, Boulassel MR, Samarani S, et al. IL-21 enhances NK cell functions and survival in healthy and HIV-infected patients with minimal stimulation of viral replication.J Leukoc Biol $2010 ; 87: 857-67$.

28. Parmigiani A, Pallin MF, Schmidtmayerova $\mathrm{H}$, et al. Interleukin-2l and cellular activation concurrently induce potent cytotoxic function and promote antiviral activity in human CD8 T cells. Hum Immunol $2011 ; 72: 115-23$.

29. Abrams D, Lévy Y, Losso M, et al. Interleukin-2 therapy in patients with HIV infection. N EnglJ Med 2009 ; 361 : 1548-59.

30. Pallikkuth $S$, Rogers $K$, Villinger $F$, et al. Interleukin-2l administration to rhesus macaques chronically infected with simian immunodeficiency virus increases cytotoxic effector molecules in T cells and NK cells and enhances $B$ cell function without increasing immune activation or viral replication. Vaccine 2011 ; 29 : 9229-38.

31. Rodrigues L, Nandakumar S, Bonorino C, et al. IL-21 and IL-15 cytokine DNA augments HSV specific effector and memory $C D 8^{+} \mathrm{T}$ cell response. Mol Immunol $2009 ; 46: 1494-504$.

32. Cui FD, Asada H, Jin ML, et al. Cytokine genetic adjuvant facilitates prophylactic intravascular DNA vaccine against acute and latent herpes simplex virus infection in mice. Gene Ther $2005 ; 12: 160-8$.

33. Hu K, Dou J, Yu F, et al. An ocular mucosal administration of nanoparticles containing DNA vaccine $\mathrm{pRSC}-\mathrm{gD}-\mathrm{IL}-21$ confers protection against mucosal challenge with herpes simplex virus type 1 in mice. Vaccine $2011 ; 29$ : 1455-62.

34. Van Leeuwen $E M$, Gamadia LE, Baars PA, et al. Proliferation requirements of cytomegalovirus-specific, effector-type human CD8 ${ }^{+} T$ cells. J Immunol $2002 ; 169: 5838-43$.

35. Van Leeuwen EM, van Buul JD, Remmerswaal EB, et al. Functional re-expression of CCR7 on CMV-specific CD8 ${ }^{+} \mathrm{T}$ cells upon antigenic stimulation. Int Immunol $2005 ; 17$ : 713-9.

36. Bolesta $\varepsilon$, Kowalczyk A, Wierzbicki A, et al. Increased level and longevity of protective immune responses induced by DNA vaccine expressing the HIV-1 Env glycoprotein when combined with IL-21 and IL-15 gene delivery. J Immunol 2006 ; 177 : 177-91.

37. Leung-Theung-Long S, Guerder S. Les cellules Th17 : une nouvelle population de cellules T CD4 effectrices pro-inflammatoires. Med Sci (Paris) 2008 ; 24 : 972-6.

38. Lambotte 0. Les patients HIV controllers. Med Sci (Paris) $2012 ; 28: 172-8$. 